\title{
DIE NEUE PAKISTANISCHE VERFASSUNG VON APRIL 1973
}

\author{
Von Helmut R. KüLZ
}

Die pakistanische Verfassungsgeschichte von 1947 bis heute ist ein getreues Spiegelbild der vielfachen und vielschichtigen Schwierigkeiten, die auch diesem nach langer Kolonialherrschaft unabhängig gewordenen Lande nicht erspart geblieben sind. Zur richtigen Einschätzung vorab ein kurzer Blick auf die anderen Teile des ehemaligen anglo-indischen Reiches. Auch in New Delhi und im unglücklichen Bangla Desh bis in unsere Tage schwerste Staats- und Wirtschaftskrisen, dern Bewältigung sich sogar noch schwieriger anzulassen scheint in Pakistan, wo - von uns aus gesehen - vom Vermächtnis der Ordnungen von Westminster, mit dem England vor rund 30 Jahren diese Gebiete in die Unabhängigkeit entließ, verhältnismäßig noch am meisten übriggeblieben ist.

Die heutige Verfassung von Pakistan ist am 10. April 1973 von der verfassunggebenden Versammlung fast einstimmig - bei nur 2 Enthaltungen von insgesamt 146 Abgeordneten - beschlossen, am 12. April 1973 vom damaligen Präsidenten, heutigen Ministerpräsidenten Bhutto bestätigt und zum 14. August $1973-\mathrm{dem}$ Jahrestag der Unabhängigkeit des Landes - in Kraft gesetzt worden ${ }^{1}$. Im Rahmen der Verfassung hat Bhutto in den seither verflossenen rund 2 Jahren zweifellos entscheidend das neue Gesicht des Landes geprägt, das sich deutlich von seinen früheren Ordnungen unterscheidet.

Die ersten 9 Jahre seiner Unabhängigkeit von 1947 bis 1956 verbrachte das Land ohne eigentliche Verfassung noch unter der Herrschaft der alten Britischen Übergangsgesetze ${ }^{2}$. Dem Staatsgründer Pakistans und großen Gegenspieler Gandhis, dem Quaid-i-Azam Mohammad Ali Jinnah, der die Unabhängigwerdung seines Landes nur um wenig länger als ein Jahr überlebte ${ }^{3}$, war es nicht mehr vergönnt, seiner Schöpfung auch noch eine Verfassung zu geben.

Die erste Verfassung Pakistans trat im Februar 1956 in Kraft; sie sah eine bi-föderale, parlamentarische Ordnung vor: bi-föderal zwischen Westpakistan mit seinen 4 Provinzen Punjab, Sind, Nordwestgrenz-Provinz und Baluchistan ${ }^{4}$ als eine Einheit („one unit“), Ostpakistan - dem heutigen Bangla Desh — als die andere Einheit, wobei die beiden Teile auch als die beiden Flügel („wings“) des Landes bezeichnet wurden. Bereits nach knapp zweieinhalb Jahren zerbrach diese Verfassung aus hier nicht weiter darzustellenden Gründen, und alle Macht ging im Oktober 1958 auf den Armeeoberbefehlshaber Ahyub Khan über; die rund 10jährige

\footnotetext{
1 Amtl. Text vom Manager of Publication Karachi herausgeg. und von der Printing Co. of Pakistan Islamabad gedruckt, 179 S., 3,50 PR. Wegen weiterer Einzelheiten zur neuen Verfassung Pakistans vgl. Munir, M., The Constitution of the Islamic Republic of Pakistan, Being Commentary of the Constitution of 1971, Law Publishing Company Lahore 1975. Die letzten Anderungen der Verfassung sind darin noch nicht mit berücksichtigt. Vgl. auch in „Pakistan, das Land und seine Menschen, Geschichte, Kultur, Staat und Wirtschaft" hrsg. v. M. Usman Malik u. Annemarie Schimmel, 1976 beim H. Erdmann Verlag, die Aufsätze von Dr. M. S. Chaudhry über "Die Politischen Parteien in Pakistan“ und von Verlag, die Aufsätze von Dr. M. S. Chaudhry über „Die Politischen Parteien in Pak
Prof. K. J. Newman über „Innen- und verfassungspolitische Probleme“, S. $207 \mathrm{ff}$. a. a. O.

2 Government of India Act 1935 i. Verb. m. d. Indian Independance Act 1947, ausführlich behandelt bei A. Gledhill, Pakistan - The Development of its Laws and Constitution, London, Stevens \& Sons, 2. Aufl. 1967, S. $60 \mathrm{ff}$.

3 Gest. am 11. September 1948; im einzelnen: Javid Iqbal, The Legacy of Quaid-i-Azam ("Großer Führer“), Lahore, Ferozons Ltd., 1967.

4 So die amtl. Schreibweise nach Art. 1, Abs. 2 der heutigen Verf.
} 
Ära Ayub Khan hatte begonnen ${ }^{5}$. Ihre ersten 4 Jahre waren reine Kriegsrechtsjahre. Mit dem Jahre 1962 trat wieder eine von Ayub Kahn erlassene Verfassung in Kraft, allerdings eine reine Präsidial- und auf die Person des Präsidenten zugeschnittene Verfassung. Ansätze zur parlamentarisch-demokratischen Willensbildung waren nur schwach vorhanden; das System der sog. „Basic Democracy“ sah unmittelbare Wahlen nur auf unterster Ebene vor. Zur Nationalversammlung wurde nur indirekt und ohne entscheidende Befugnisse für das Parlament gewählt. Immerhin war unter der Verfassung von 1962 schon weitgehend die richterliche Unabhängigkeit wieder gewährleistet gewesen ${ }^{6}$. Im März 1969 ging auch die Ära Ayub Khan unter erheblichen Erschütterungen des ganzen Landes zu Ende und alle Macht wiederum unter Kriegsrecht auf den Armeeoberbefehlshaber Yayah Khan über. Eine im März 1970 von ihm erlassene „Legal Framework Order“ - Verfassungsrahmengesetz - sollte zwar die Rückkehr zu einer normalen demokratisch-parlamentarischen Ordnung vorzeichnen ${ }^{7}$, endlich auch dem bevölkerungsmäßig stärkeren Ostpakistan die bisher versagte unbedingte Stimmengleichheit bringen - „one man - one vote“ - mit zweifelsfrei zu erwartendem Übergewicht des Ostens in einer neuen Nationalversammlung, die so auch am 7. Dezember 1970 gewählt wurde, aber in ihrer Gesamtheit nie zusammengekommen ist. Die Ostprovinz trat mit entscheidender Unterstützung Indiens in den Sezessionskrieg ein, der das Ende des damaligen pakistanischen Zwei-Flügel-Staates brachte, auch das Ende der Herrschaft des als Staatsmann wie Feldherrn gleichermaßen glücklosen Yayah Khan.

Die verfassunggebende Versammlung trat nur mit den schon erwähnten 146 Abgeordneten aus den 4 Westprovinzen zusammen; die vom jetzigen Ministerpräsidenten Bhutto neu gegründete Pakistan Peoples Party - Pakistanische Volkspartei - errang mit 81 Abgeordneten die eindeutige Mehrheit in dieser Versammlung ${ }^{8}$ - die Ära Bhutto hatte ihren Anfang genommen.

Die neue Verfassung, die aus dieser Versammlung hervorging, ist mit $12 \mathrm{Ab}$ schnitten und 280 Artikeln nebst verschiedenen Anlagen fast doppelt so umfangreich als unser Grundgesetz und bietet schon damit das Bild einer besonders ausgefeilten und sorgfältigen Ordnung9. Überall treffen wir dabei, wie schon angedeutet, auf die vertrauten Bestimmungen einer pluralistischen parlamentarischen und föderalen Demokratie mit angemessenen Rechtsgarantien, sowohl für die festgelegten Strukturen wie auch für die in der Verfassung gewährleisteten Grund- und Menschenrechte. Grundlage der Staatsordnung sind unmittelbare, freie, geheime und gleiche Wahlen nach dem Mehrheitswahlrecht, wahlberechtigt sind alle $\mathrm{Pa}$ kistani, auch Frauen, über 18 Jahre (Art. 51). Es werden sowohl eine Nationalversammlung mit 200 Abgeordneten (Art. 51) als auch Provinzialversammlungen mit einer nach der Bevölkerungszahl der 4 Provinzen abgestuften Größe gewählt (Art. 106). Zur Nationalversammlung treten bis auf weiteres 10 zusätzliche weibliche Abgeordnete (Art. 51, Abs. 4); die Provinzialversammlungen weisen jeweils

5 Mohammed Ayub Khan, Erinnerungen und Bekenntnisse (engl. Titel: „Friends Not Masters“), Tübingen/ Basel, Horst Erdmann Verlag 1968.

6 Näheres bis dahin Gledhill a. a. O.

$7 \mathrm{Im}$ einzelnen H. R. Külz, Pakistan auf dem Wege zu einer neuen Verfassung, in Verfassung und Recht in Übersee, 1970 (3. Heft), S. $357 \mathrm{ff}$.

8 Genaue UUbersicht mit Erläuterungen und weiteren Nachweisen im Archiv der Gegenwart ("Keesing's Archiv“) 1971 , S. $1623 \mathrm{ff}$

9 Eine erste umfassende Würdigung der Verf. gibt Munir D. Ahmed, The Permanent Constitution of Pakistan, in Orient, 1973 (Nr. 3), S. $118 \mathrm{ff}$. 
1-3 besondere Sitze für die religiösen Minderheiten auf (Art. 106, Abs. 3). Alle 4 Provinzialversammlungen wählen sodann gleichermaßen je 14 Abgeordnete in den Senat (Art. 59), die zweite Kammer des Landes, die etwa unserem Bundesrat vergleichbar ist. Nationalversammlung und Senat zusammen bilden das in bestimmten Fällen zuständige Gesamtparlament des Landes (Art. 50). Aus Nationalversammlung und Provinzialversammlungen gehen die parlamentarisch verantwortlichen Regierungen des Bundes und der Provinzen hervor (Art. 90 ff. und Art. $131 \mathrm{ff}$.), mit einem Ministerpräsidenten an der Spitze der Bundesregierung und 4 Chefministern ("Chief Ministers") an der Spitze der Provinzialregierungen. Bei allfälligem Mißtrauensvotum gegen den Ministerpräsidenten folgt die Verfassung dem deutschen Vorbild des konstruktiven Mißtrauensvotums, so daß es nur bei gleichzeitiger Neuwahl eines anderen Ministerpräsidenten zum Zuge kommt (Art. 96, Abs. 2), allerdings mit der interessanten, den absoluten Fraktionszwang sichernden Variante, daß bis auf weiteres Stimmen, die abweichend von der Mehrheit einer Fraktion abgegeben werden, außer Betracht zu lassen sind (Art. 96, Abs. 5). Das Staatsoberhaupt des Landes ist ein vom Gesamtparlament - Nationalversammlung und Senat - auf 5 Jahre zu wählender und einmal wieder wählbarer Staatspräsident (Art. 41); an der Spitze der Provinzen stehen vom Präsidenten zu ernennende Gouverneure (Art. 101). Für die Abgrenzung der Zuständigkeiten zwischen Bund und Provinzen unterscheidet die Verfassung wie bei uns ausschließliche Bundeszuständigkeiten, zwischen Bund und Ländern konkurrierende Zuständigkeiten und ausschließliche Landeszuständigkeiten (Art. 70 ff. i. Verb. mit Anhang 4: „Legislative Lists“). Auswärtige Angelegenheiten, Verteidigungsangelegenheiten, das Währungswesen und der Außenhandel gehören zu den ausschließlichen Bundeszuständigkeiten. Einige besonders interessante Punkte seien noch kurz ein wenig im einzelnen behandelt.

Zunächst die heikle Frage, wie die Verfassung mit der schon kurz gestreiften Sezession der Ostprovinz fertig geworden ist. Am Beginn der neuen Ordnung stand zunächst - in uns wohlvertrauten, durchaus verständlichen und an sich auch voll berechtigten Denkansätzen - die Auffassung von der Illegalität der gewaltsamen Teilung des Landes und Abtrennung der Ostprovinz, m. a. W. der Grundsatz vom de jure Fortbestand eines gesamten Pakistan mit einem unbedingten Wiedervereinigungsgebot. Es war in Art. 1, Abs. 3 wie folgt zum Ausdruck gekommen:

Die Verfassung wird in geeigneter Weise geändert werden, um dem Volke der Provinz Ost-Pakistan - sobald die derzeitige ausländische Agression in dieser Provinz und ihre Folgen beseitigt sein werden - die Möglichkeit zur Vertretung in den Angelegenheiten des Bundes einzuräumen,

eine etwas an den Schlußartikel 146 unseres Grundgesetzes gemahnende Vorschrift. Nach der völkerrechtlichen Anerkennung von Bangla Desh durch Pakistan ist indessen dieser Artikel ersatzlos gestrichen worden ${ }^{10}$, so daß ein Wiedervereinigungsgebot aus der Verfassung nicht mehr abzuleiten ist.

Ein weiteres Wort zur gegenwärtigen Verfassungslage und Verfassungsverwirklichung im Hinblick auf das Verhältnis zwischen Regierung und Opposition. Daß von einem Einparteiensystem und einer Einparteienherrschaft seitens der Pakista-

10 Vgl. dazu - und zu anderen Verfassungsfragen des Landes - W. Wengler, Richter, Revolution, Notstand und kein Ende - Oder: Kelsen in der Dritten Welt, in Archiv des Offentlichen Rechts, 1974, (Heft 3), S. $464 \mathrm{ff}$., insbes. S. 475 . 
nischen Volkspartei Bhuttos nicht die Rede sein kann, war schon oben durch Bezugnahme auf die Sitzverteilung in der verfassunggebenden Nationalversammlung klargestellt worden, in der die Regierungspartei zwar über eine entscheidende Mehrheit verfügt, aber keineswegs die allein maßgebende Partei ist. Demgegenüber werden in der westlichen Presse immer wieder unrichtige und entstellende Angaben verbreitet, als ob Bhutto gewaltsam oder mit sonst unzulässigen Mitteln alle Opposition zum Schweigen bringen wolle. Den besten Gegenbeweis hat in jüngster Zeit wieder die am 20. Oktober dieses Jahres in Lahore, der Hauptstadt des Punjab, abgehaltene Nachwahl erbracht. Bei ihnen hatte der aus der PPP ausgetretene frühere Gouverneur der Provinz, Ghulam Mustafa Khar, der Regierungspartei einen überaus aktiven und erbitterten Kampf geliefert, bei dem er sich aber nicht durchsetzen konnte; um rund 15000 Stimmen war er hinter seinem Gegenkandidaten zurückgeblieben. Die Neue Zürcher Zeitung, gewiß ein unverdächtiger Beurteiler, bemerkt dazu zutreffend folgendes:

„Seine - Kahrs - bereits vor dem Urnengange aufgestellte Behauptung, die Wahlen würden von Regierungsseite gefälscht werden, kann er kaum glaubhaft aufrechterhalten ${ }^{11 . "}$

So müssen sich die Oppositionsparteien weiterhin in der Stellung einer Minderheit sehen, aber einer tatsächlich echten, nicht etwa einer manipulierten Minderheit, im Gesamtparlament ebenso wie insbesondere in der mit zwei Dritteln aller Wähler besonders gewichtigen Punjab-Provinz.

In den an Afghanistan grenzenden Bergprovinzen, der Nordwestgrenz-Provinz und Beluchistan, liegen die Parteistrukturen etwas anders. Hier hatte Bhutto zunächst die unter seinen Vorgängern verboten gewesene National Awami Party (NAP) des einflußreichen und mächtigen Landesfürsten Khan Abdul Wali Khan wieder völlig unbehindert und bedingungslos zugelassen, so daß sie sich nicht ohne Erfolg an den Wahlen gegen die Regierungspartei des Bundes beteiligen konnte. Zusammen mit der Jamiat-ul-Ulema, einer konservativen Muslim-Partei, konnte sie demgemäß auch die Provinzregierungen bilden, darüber hinaus war ihr und der Jamiat-ul-Ulema angeboten worden, je einen Minister in die Bundesregierung zu entsenden. Die NAP begrüßte diese Entwicklung und sicherte der Bundesregierung auf ihre Vorschläge loyale Zusammenarbeit $\mathbf{z u}^{12}$. Aber Wali Khan, der tatsächliche Beherrscher der Partei, sagte sich von allen Absprachen mit der Regierung plötzlich wieder los. Von der NAP zu verantwortende Sabotageakte und Gewalttätigkeiten aller Art, Bombenattentate, Ermordungen anders gesinnter, auf seiten der Bundesregierung stehender Persönlichkeiten des öffentlichen Lebens und blutige örtliche Stammeskriege setzten ein. Immer wieder unverhohlener wurde dabei von der NAP auch - von Afghanistan und Indien offen unterstützt - der Abfall der Provinzen von Pakistan als letztes politisches Ziel verkündet. Bei alledem handelte es sich aber um nichts weniger als den Freiheitskampf unterdrückter Stämme gegen eine rücksichtslose Zentralinstanz; Sprache sowie Sitten und Gebräuche der Bergstämme sind von der Zentralregierung niemals beeinträchtigt worden, außer daß sie - seit jeher - die fortdauernden mörderischen Blutfehden zu unterbinden bestrebt ist, die die Siedlungen dieser Provinzen von alters her heimsuchen und

11 Neue Zürcher Zeitung vom 21. 10. 1975, S. 4: „Wahlniederlage der Pakistanischen Opposition - Prestigeerfolg Bhuttos. ${ }^{\alpha}$

12 S. o. Anm. 8, insbes. die dort über die NAP mitgeteilten Einzelheiten. Vgl. auch „The Tripartite Accord March 6, 1972, Basic Documents and Background Material. 
dezimieren ${ }^{13}$. Bei der NAP handelt es sich, völlig abseits von solchen Problemen, eindeutig um eine für die Interessen des Großgrundbesitzes eintretende Feudalpartei, deren Ziel die Verhinderung der von Bhutto und seiner Volkspartei erstrebten Reformen, vor allem der Land- und Bodenreform war und ist, unter Erhaltung archaischer Herrschaftsformen der Vergangenheit, wie sie gewiß in einem sich fortschrittlich entwickelnden Lande keinen Platz mehr haben möge. Es verfügte denn auch die NAP in den beiden Bergprovinzen keineswegs über eine alle anderen Richtungen erdrückende und eindeutige, sondern nur über eine sehr relative Mehrheit, in der letzten Provinzialversammlung von Beluchistan z. B. nur über 8 von insgesamt 21 Sitzen, gegen immerhin 7 Abgeordnete von Bhuttos $\mathrm{PPP}^{14}$. Überdies dürfte ein erheblicher Teil der für die NAP abgegebenen Stimmen weniger auf freier Willensentscheidung der Wähler als auf patriarchalischen und kleinbäuerlichen Abhängigkeitsverhältnissen beruhen, die sich bisweilen sehr deutlich in gemeinsamen Wahlmärschen der Dörfer zwecks gemeinsamer Stimmabgabe abgezeichnet haben ... Die Bundesregierung hatte schließlich, wenn anders sie Ruhe und Ordnung im Lande wiederherstellen und die Unversehrtheit des Staatsgebietes sichern wollte, gar keine andere Wahl, als im Februar 1975 die NAP wieder aufzulösen und $\mathrm{zu}$ verbieten; auch keine unserer westlichen Verfassungsordnungen läßt sich widerstandslos von staatsfeindlichen Parteien zerstören oder unterhöhlen. Das Verbot und die Auflösung der NAP wurden im übrigen nicht etwa unter wieder eingeführtem Kriegsrecht oder sonst nach willkürlichem Ausnahmerecht durchgeführt, sondern streng in den dafür in der Verfassung vorgesehenen Formen mit Anrufung des Obersten Gerichtshofs des Landes. Nach sorgfältig vorbereiten, mehrmonatlichen und völlig unparteiischen Verfahren hat gerade dieser Tage, am 30. Oktober 1975, das Plenum des Obersten Gerichtshofs einstimmig das Verbot der NAP bestätigt ${ }^{15}$. Die demnächst zu erwartenden Gründe des Urteils der unbestritten anerkannten und unabhängigen höchsten Richter des Landes werden jeden Unbefangenen von der Richtigkeit und Gerechtigkeit der Entscheidung überzeugen und damit endgültig die Vorwürfe willkürlicher Unterdrückung einer unbequemen Oppositionspartei entkräften, zumal die anderen Oppositionsparteien in den in Frage kommenden beiden Provinzialversammlungen davon in keiner Weise mitbetroffen sind. Schon jetzt ist die überaus sachliche und gründliche Anklageschrift lesenswert ${ }^{16}$, die Ende Juni d. J. der Generalstaatsanwalt des Landes dem Gerichtshof vorgetragen hat und die keineswegs nur eine nüchterne und einseitige Zusammenstellung von allerhand Belastungsmaterial enthält. Die ganze politische und Parteiengeschichte der Bergprovinzen mit vielen farbigen Schlaglichtern wird darin aufgerollt, auch nicht ohne Verständnis für manche tragischen und dramatischen Seiten der dortigen Entwicklungen und ihrer Träger; vielfach sehen wir, wie sich persönliche, Sippen- und Stammesschicksale mit dem Wohl und Wehe des Landes im Ganzen zu schier unlösbar scheinenden Knoten verwickelt haben, die nun eben nicht anders als durch ein Verbot der NAP entwirrt werden konnten.

\footnotetext{
13 Prof. Mohammad Ali, Vizekanzler der Universität Peshavar, „. . And Then The Pathan Murders ${ }^{\alpha}$, Peshavar, University Book Agency, 1966.

14 Weißbuch der Pakistanischen Regierung über Baluchistan „Main Points ${ }^{\alpha}$, 19. 10. 1974, S. 17.

15 Times (London) vom 31. 10. 1975, S. 8: "Pakistan "Court Upholds Bhutto Government's Ban on Opposition NAP ${ }^{\alpha}$, mit wichtigen Erläuterungen zu der vorliegenden Entscheidung.

16 Yayayh Bakhtiar, Opening Address in the Supreme Court of Pakistan in the Islamic Republic of Pakistan vs. Abdul Wali Khan, an Dissolution of NAP, Rawalpindi, Juni 1975 (55 Seiten).
} 
Für den westlichen und europäischen Betrachter ist schließlich der stark islamische Einschlag der Verfassung bemerkenswert. Schon der Überschrift „Im Namen Allah's des Wohltätigen und Gnädigen “ - und in der Präambel der Verfassung wird der "Allmächtige Allah als einziger Herr des Universums“ angerufen und insbesondere den Muslims des Landes die Ordnung ihres Lebens in „Übereinstimmung mit Lehre und Geboten des Islam nach Maßgabe des heiligen Koran und der Sunnah" versprochen. Auch die Beibehaltung des Namens „Islamische Republik" ist kennzeichnend (Art. 1). Die völlig neue Bestimmung des Islam zur Staatsreligion (Art. 2) und die Bestimmung, daß Präsident und Ministerpräsident Muslims sein müssen (Art. 41, Abs. 2 und Art. 91, Abs. 2) machen deutlich, daß es nicht nur beim Namen einer Islamischen Republik bewenden soll. In gleicher Richtung gehen die besonders streng gefaßten Amtseide des Präsidenten und Ministerpräsidenten (3. Anhang zur Verfassung), die ihren Glauben an Koran und Sunnah sowie an Mohammed als den unbedingt letzten Propheten und an das Jüngste Gericht beschwören müssen; auch die Bundesminister und Provinzialminister müssen - etwas weniger streng - auf die islamische Ideologie als Grundlage der Entstehung Pakistans schwören. Darüber hinaus enthält Abschnitt 9 der Verfassung: „Islamic Provisions" eine Reihe weiterer Einzelvorschriften mit der Grundregel (Art. 227), daß das gesamt bestehende Recht in Übereinstimmung mit den Geboten des Islam gebracht werden soll, wie sie in Koran und Sunnah niedergelegt sind. Ebenso darf kein kün itiges Gesetz diesen Geboten widersprechen. Die Einhaltung dieser Gebote ist ein $2 m$ Islamischen Rat („Islamic Council“, Art. 228) anvertraut, der vom Präsidenten ernannt wird und unter dem Vorsitz eines der obersten Richter des Landes steht. Bei Zweifeln an der Verträglichkeit eines Gesetzes mit dem Islam kann der Präsident, jeder Provinzgouverneur oder $2 / 5$ der Mitglieder der National- oder einer Provinzialverfassung die Stellungnahme des Rates herbeiführen (Art. 229), freilich nur mit der Folge, daß bei Bejahung solcher Zweifel durch den Rat eine nochmalige Beratung des Gesetzes stattzufinden hat, bei dringendem öffentlichen Interesse auch nicht mit der Folge einstweiligen Aufschubs eines solchen Gesetzes (Art. 230 Abs. 2 und 3); dem Rat steht also nicht einmal ein unbedingtes suspensives Veto zu. Daneben hat er aber noch allgemeine Empfehlungen zur besseren Verwirklichung der Gebote des Islam zu geben (Art. 230 Abs. 1). Allgemeine Bürgerrechte u. a. m. sind unabhängig vom Glaubensbekenntnis gewährleistet (Art. 2C ff., Art. 27), ebenso allgemeine Glaubens- und Bekenntnisfreiheit, bei der allerdings „the Glory of Islam" gebührend berücksichtigt werden muß (Art. 19). Alle diese Bestimmungen waren bei Beratung der Verfassung heftig umstritten. Von konservativ-islamischer Seite waren noch schärfere Verpflichtungen auf die Gebote des Koran vorgeschlagen worden, insbesondere ein unbedingtes Veto des Islamischen Rates gegen gesetzgeberische Verstöße gegen den Ko$\operatorname{ran}^{17}$. Andererseits hatte Bhuttos Volkspartei sehr viel weniger Verpflichtungen religiöser Art in der Verfassung verankert sehen wollen. Sie hat schließlich bis zu einer gewissen Grenze nachgegeben, um auch die konservativen Kräfte, insbesondere in den Bergprovinzen, zur Zustimmung für die Verfassung zu bewegen und um ihr Entstehen nicht von vornherein erbitterten Glaubenskämpfen auszusetzen, auch wenn dadurch auf der anderen Seite scharfe Kritik an allen diesen Bestimmungen

17 Vgl. z. B. den Verf.-Entwurf des Prof. und früheren High-Court-Richters Abdul Hamid, Peshawar, „Draft of New Constitution of Pakistan 1971, Karachi (Hamdrad National Foundation Pakistan), Part 9 ("Islamic Provisions ${ }^{\alpha}$ ), Art. 193 ff. 
ausgelöst worden ist, die als „mittelalterliche Relikte der Vergangenheit“ bezeichnet wurden, für die es schwierig sein werde, „den Nachkommen der heutigen Generation eine plausible Erklärung $\mathrm{zu}$ geben ${ }^{18 “ . ~ M a n ~ w i r d ~ g l e i c h w o h l ~ s a g e n ~}$ müssen, daß der gefundene Mittelweg der inneren Geschlossenheit des Landes sehr zugute gekommen ist und daß der starke islamische Einschlag der Verfassung auch nach außen hin bedeutende Wirkungen entfaltet hat. Die von Bhutto im Jahre 1973 in die berühmte Badschaj-Moschee von Lahore einberufene umfassende Islam-Konferenz - eine großartige ökumenische Veranstaltung dieses Glaubensbereiches, an der die Führer von nahezu 600 Millionen Muslims aus aller Welt teilnahmen, von Marokko bis Indonesien - wäre so ohne das betont islamische Staatsverständnis des Landes wohl kaum zustande gekommen; auch der politischen Stellung Pakistans in der gesamten islamischen Welt und darüber hinaus ist die Konferenz zugute gekommen. Sie erfüllte sichtbar den Verfassungsauftrag in Art. 40 der dem Staat „die Erhaltung und Stärkung der auf die islamische Einheit gegründeten brüderlichen Beziehungen zwischen den Muslims aller Länder" auferlegt ${ }^{19}$.

18 Detlev H. Khalid "The embarrassing Islamic Provisions ${ }^{\alpha}$, in Outlook, Karachi, Bd. 2 v. 28. 4. 1973, No. 4.

19 Interessant, daß a. a. O. außerdem nur noch die Unterstützung der gemeinsamen Interessen der Völker Asiens, Afrikas und Latein-„Amerikas" ${ }^{\alpha}$ - also der Dritten Welt - als Verfassungsrichtlinie ausgesprochen ist. 
The prospects for development of the large group of DC, which are poor in resources, is examined in an analysis of the AKP-Treaty of Lomé (1975). In spite of several trade concessions granted by the European Community, the accelerated specialization of African countries for exports of raw materials, vegetables, fruits and of labour-intensive industrial products (for European markets) seems not suitable to solve the basic problems of structural dependence and underdevelopment.

\section{The New Constitution of Pakistan of April 1973}

\section{By Helmut R. Külz}

The author is beginning with a short history of the being, third Constitution of Pakistan, almost unanimously accepted by a freely elected National Assembly on the 10. April 1973 - the start of the Bhutto-aera. The main features of the Constitution as an instrument of a pluralistic, parliamentary and federal system are then being presented (direct, free, equal and secret elections for a National Assembly and four Provincial Assemblies, with special seats for women and religious minorities, a Senate as a second chamber; central and provincial governments; exact definitions of federal and provincial powers; election of a President of Pakistan and nomination of Governors for the Provinces; judicial independence and guarantees). A remarkable first amendment of the Constitution is referred to (possibility of re-unification with Bangla Desh no longer being inserted). In some detail the various "Islamic Provisions" are being expounded (Islam as State Religion, all Laws in conformity with Holy Khoran and Sunnah; special organ - "Islamic Council" - for supervision of this conformity, although without real power to enforce dissenting views against decisions of legislative bodies), all those islamic provisions being a compromise between stronger religious tendencies and the opposite tendency in direction of a merely secular constitution. On the other hand the political relevance and importance of all these islamic provisions of the Constitution is underlined, also for the foreign policy of Pakistan (oecumenic Islamic Conference in Lahore in 1975, enhancing considerably $\mathrm{Pa}-$ kistan's and Bhutto's prestige in the islamic world and beyond). In conclusion the author is dealing with the relations between Government and Opposition, repudiating strongly and in detail the common prejudice of arbitrary supression of all opposition in Pakistan. The ban of the National Awamy Party is being explained as a well-founded action against a subversive, anti-constitutional organization eventually engaged in terrorism and bloodshed, facts established beyond doubt in long and unbiassed court proceedings. 